\title{
The NICA Complex and the MPD experiment at JINR: status and physics potential
}

\author{
Adam Kisiel ${ }^{1,2, *}$ \\ ${ }^{1}$ Joint Institute for Nuclear Research, Joliot-Curie 6, 141980 Dubna. Moscow Region, Russia \\ ${ }^{2}$ Faculty of Physics, Warsaw University of Technology, Koszykowa 75, 00-662 Warsaw, Poland
}

\begin{abstract}
The Multi-Purpose Detector is under construction at the Joint Institute for Nuclear Research in Dubna, as part of the NICA Accelerator Complex. The MPD Collaboration aims to study the phase diagram of QCD matter at maximum baryonic density, determine the nature of the phase transition between the deconfined and hadronic matter and search for the critical point. The combination of significant luminosity at NICA, collider geometry of the experiment and collision energies $\sqrt{s_{\mathrm{NN}}}$ spanning the range of 4 to $11 \mathrm{GeV}$, where the transition from baryon to meson dominated matter is expected will allow MPD to provide unique insight into these questions. The status of the assembly of the detector in MPD Hall, as well as the progress in the production of MPD subdetectors will be reported on.

The design physics performance of the detector will be discussed with the emphasis on the measurements in the strangeness sector. Tracking and particle identification procedures will enable precise determination of spectra of identified hadrons, including hyperons and hypernuclei, with the possibility of differential measurements and total yield extraction. The quality of directed and elliptic flow determination will be discussed, with comparison to model expectations. The sensitivity of event-by-event fluctuations to the nature of the phase transition and the presence of a critical point will be given. The femtoscopic analysis for charged pions and kaons has shown sensitivity to first order and cross-over phase transitions in EoS seen for the vHLLE model. All those studies will be put in context of addressing outstanding questions on the properties of the region of the QCD phase diagram probed by heavy-ion collisions in the NICA energy range.
\end{abstract}

\section{Introduction}

The Nuclotron-base Ion Collider fAcility (NICA) is a major science project realized by the Joint Institute for Nuclear Research (JINR). Its aims to investigate phase diagram of QCD matter in the region of maximum baryonic density by studying (heavy-)ion collisions in the energy range from 2.5 to $11 \mathrm{GeV} / \mathrm{u}$. The Baryonic Matter at Nuclotron (BM@N) and MultiPurpose Detector (MPD) experiments will collect data at the facility. This work describes the status of construction of NICA and the MPD experiment, and gives a brief summary of its scientific goals.

\footnotetext{
*e-mail: Adam.Kisiel@jinr.ru
} 


\section{NICA and MPD construction status}
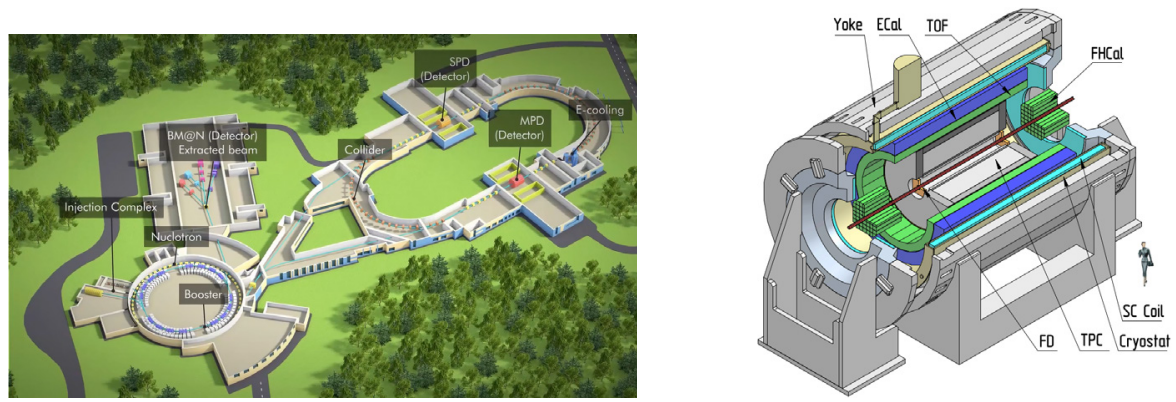

Figure 1. (Left) The schematic view of the NICA complex. (Right) The setup of the MPD apparatus in the Stage I of NICA.

The ion beams for NICA (schematically shown in Fig. 1) are provided by two injection complexes. The KRION source coupled to the HiLac linac produce light ions up to carbon. Dedicated sources with LU-20 linac produce heavier ions, including krypton, xenon and bismuth. Both sources are commissioned and operating. Nuclotron is a circular accelerator based on fast-cycling superconducting magnets, able to accelerate ions from protons up to bismuth with maximum kinetic energy of $3.8 \mathrm{GeV} / u$ for $\mathrm{Au}$ ions. It is in operation since 1993. Booster is an auxiliary accelerator, needed for pre-acceleration of heavy ions up to $600 \mathrm{MeV} / u$. It has been put into operation in December of 2020. The commissioning of the full Booster and Nuclotron complex is planned for the end of 2021 and beginning of 2022. Once completed, they will provide beams of ions to the fixed-target area, where the Baryonic Matter at Nuclotron (BM@N) experiment is operating. Physics runs with beams of $\mathrm{Fe} / \mathrm{Kr} / \mathrm{Xe}$ are planned in 2022 for this experiment. In parallel the civil construction and production of magnets is progressing for the NICA collider. Installation of equipment is planned in stages through 2021 and 2022, the commissioning is expected in late 2022 and first beams will be provided in 2023. NICA is a racetrack-shaped collider, with $503 \mathrm{~m}$ of circumference. Two experimental halls are located in two straight sections. NICA is designed to provide $\mathrm{Au}+\mathrm{Au}$ collisions in the center-of-mass energy $\sqrt{s_{\mathrm{NN}}}$ range of 4 to $11 \mathrm{GeV}$. Luminosity at maximum energy is $10^{27} \mathrm{~cm}^{-2} \mathrm{~s}^{-1}$. NICA complex can accelerate ions from protons and deuterons (possibility for polarized beams will be added in the Stage II of project preparation) through light $(\mathrm{C})$ and intermediate (Ar, Fe, $\mathrm{Kr}, \mathrm{Xe}$, and others) to heavy (Au, Bi) ions. Therefore, a complete energy vs. system size scan is a possible physics program at NICA. In 2023 $\mathrm{Bi}+\mathrm{Bi}$ collisions at $\sqrt{s_{\mathrm{NN}}}=9.2 \mathrm{GeV}$ are planned for initial NICA operation. Luminosity is expected to be approximately $10^{25} \mathrm{~cm}^{-2} \mathrm{~s}^{-1}$ with a few months of running time. 2024 will be dedicated for achieving $\mathrm{Au}+\mathrm{Au}$ collisions with maximum energy of $11 \mathrm{GeV}$, with routine physics operation foreseen after reaching this goal.

The main physics experiment at NICA is the Multi-Purpose Detector (MPD), which will be operating at the collider. In 2018 an international scientific collaboration of MPD has been established. Currently it is composed of 42 institutes from 12 countries, as well as JINR as a host institution. The collaboration will be operating the MPD apparatus, which is shown schematically in Fig. 1 (right). Its designated position is the MPD Hall, which is located at the northern straight section of the NICA collider. Since 2020 the building has been available for MPD activities. 
The construction of the apparatus began in 2020. The outer magnet yoke has been fully assembled with satisfactory mechanical precision. Uniform magnetic field up to $0.57 \mathrm{~T}$ (with the default setting of $0.5 \mathrm{~T}$ ) will be provided by the superconducting solenoid, which has been manufactured by the ASG company in Italy. It has been delivered to JINR in November of 2020 and has been placed inside the magnet yoke in July of 2021. The completion of the assembly, commissioning and magnetic field measurements are foreseen for the reminder of the year. The magnet yoke will also serve as a mechanical fixing point for the dedicated carbon fiber support structure, which will house other detectors. It will be installed in 2022.

Located closest to the beam, the Time Projection Chamber (TPC), a $3.4 \mathrm{~m}$ wide, barrel shaped gaseous tracking detector will determine the charged particles' trajectories and their specific energy loss $\langle\mathrm{d} E / \mathrm{d} x\rangle$. Maximum event rate for the TPC is $7 \mathrm{kHz}$. All components of the TPC vessel are ready and the production of the read-out chambers is progressing at JINR. The Time-of-Flight (ToF) detector will surround the TPC. Its Multigap Resistive Plate Chambers (MRPC) will measure the arrival time of charged particles with time resolution of $50 \mathrm{ps.}$ Together, TPC and TOF will allow for discrimination between pions and kaons up to $p_{\mathrm{T}}$ of $1.5 \mathrm{GeV} / c$ and mesons from protons up to $2.5 \mathrm{GeV} / c$. Significant sample of MRPCs is already produced at JINR. TPC and ToF will be installed in 2022. The Electromagnetic Calorimeter (ECal) is the outermost central barrel detector. Its main goal is to measure the energy of photons and electrons produced in the collision. It is composed of shashlyk-type sampling calorimeters with plastic scintillators, light collected by WLS (Wave Length Shifting) fibers and MAPD (Micro-Pixel Avalanche Photo Diode) read-out. 38400 towers are arranged in the projective geometry in a barrel around ToF. The design energy resolution is $5 \%$ at $1 \mathrm{GeV} / \mathrm{c}$ momentum. ECal is produced jointly by Russian and Chinese collaborators. Roughly half of ECal will be installed in 2022 with the remaining modules added in the following years.

In the forward region, the Fast Forward Detector (FFD) based on fast Cherenkov modules, placed $140 \mathrm{~cm}$ from the interaction point around the beam pipe on either side, will provide level-0 trigger and start time information for the ToF. The Forward Hadronic Calorimeter (FHCal) will be located near the magnet end caps. On each side it will contain 44 sampling calorimeter modules, capable of delivering information on the energy deposited by spectator nucleons and fragments. It will allow for the determination of the event centrality as well as the orientation of the event plane at large rapidities. FFD and FHCal will be installed in 2022. The overall commissioning of the MPD detector is foreseen for late 2022 and will include calibration runs with cosmic muons. A dedicated MPD Cosmic Ray Detector (MCORD) will be used for triggering in this task. It may also be added to the MPD setup later, possibly installed on the outside of the magnet yoke.

\section{The MPD experiment physics performance and prospects}

The QCD phase diagram is shown schematically in the left panel of Fig. 2. The MPD experiment aims to characterize this diagram, in particular in the area of moderately large temperatures and large baryochemical potential. This region of the diagram has not been fully explored so far. The investigation will be carried out by performing an collision energy scan in the full NICA range of $\sqrt{s_{\mathrm{NN}}}$ from 4 to $11 \mathrm{GeV}$. The accelerator complex also allows for collision system size scan. This gives possibility for a doubly differential investigation of the onset of deconfinement. In the region of temperatures strongly exceeding the value of $160 \mathrm{MeV}$ (at which the deconfinement is expected to occur), as well as baryochemical potential approaching zero the experiments at RHIC (STAR, PHENIX, BRAHMS and PHOBOS) and LHC (ALICE, CMS and ATLAS) have performed an extensive search for deconfined matter. The production of Quark Gluon Plasma was observed at these conditions, and the transition back to hadronic matter was determined to be of the cross-over type. Going towards larger baryon 

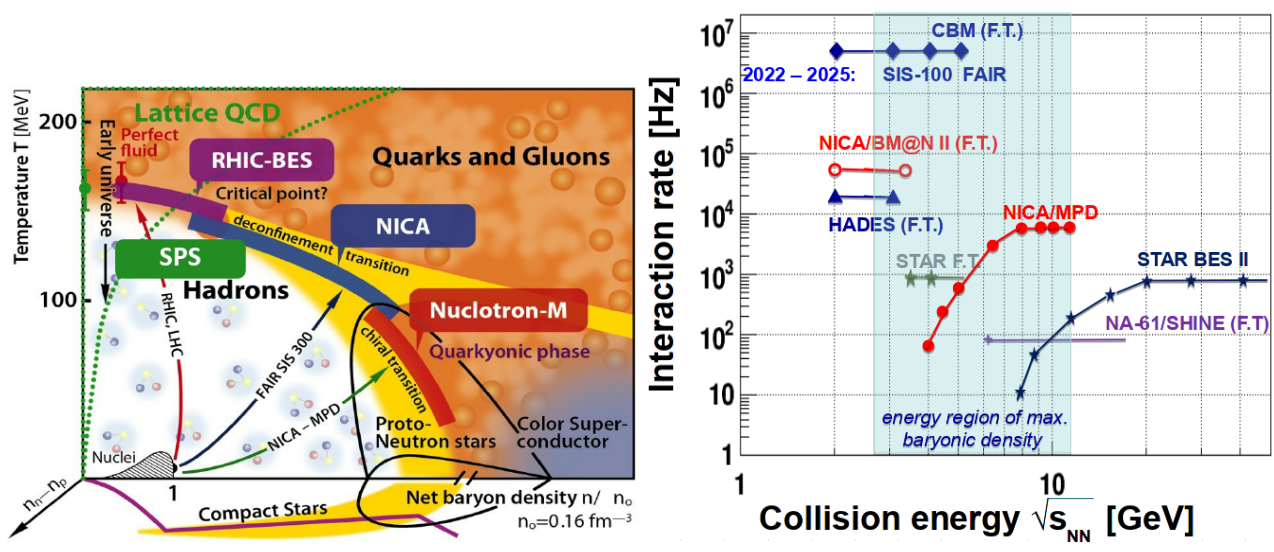

Figure 2. (left) The schematic view of the QCD Phase diagram. (right) The collision energies and expected interaction rates of currently ongoing and planned experimental programs.

densities theoretical calculations point towards the existence of first-order phase transition between QGP and hadronic matter, as well as the existence of the critical end-point in the diagram. The search for this point remains one of the major goals of the heavy-ion collision program at collision energies below $100 \mathrm{GeV}$. The experiments at SPS, the Beam Energy Scan (BES) program conducted by STAR at RHIC, as well as investigations at lower energies at GSI/FAIR and elsewhere participate in this effort. So far no conclusive experimental evidence for the existence of this critical point has been found.

MPD at NICA will provide unique coverage of collision energies and collision system sizes at large intensities with respect to other facilities operating at similar energies, as shown in the right panel of Fig. 2. Moreover, the MPD experiment is offering collider geometry in this region. This provides large acceptance, which varies only slightly with the change of the collision energy. Therefore, NICA will provide competitive experimental conditions for the exploration of the phase diagram area of interest. The $\sqrt{s_{\mathrm{NN}}}$ range of $4-11 \mathrm{GeV}$ for heavy-ion collisions allows to explore the area of maximum baryon density. The discovery potential at NICA is unique and complementary to similar scientific programs at lower energies at FAIR as well as at higher energies at SPS and RHIC BES.

The first-day physics program at MPD will focus on several key observables. The total charged track multiplicity will be measured in the TPC. In combination with the recording of the energy deposit in FHCal they enable determination of the event centrality. A Modified Wounded Nucleon, also known as MC-Glauber [1] will be employed to associate these experimentally determined quantities to the geometrical parameters, which are necessary for theory comparisons, such as the values of the impact parameter, number of participants and spectators. The careful study of nuclear fragments will also be performed. The energy density reached in the collision can be inferred from the multiplicity density per unit of pseudorapidity [2]. Its measurement will show, whether densities needed for the creation of QGP are reached in the collisions at NICA. The transverse energy density, which is related to the internal pressure in the extremely dense matter produced in collisions of heavy-ions [3] will also be measured when the particle identification will be employed.

The particle/anti-particle production ratio for identified hadrons is directly related to key aspects of the system evolution, such as the charge transport mechanism in the collision. The ratio can be measured for particle types identified in MPD, such as $\mathrm{p} / \overline{\mathrm{p}}, \mathrm{K}^{+} / \mathrm{K}^{-}$, and 
$\pi^{+} / \pi^{-}$. Provided sufficient statistics is collected, the ratios can also be determined for heavier baryons, for example $\Lambda / \bar{\Lambda}$. After extrapolation to full $4 \pi$ acceptance, total yields of various particle types, including the ones reconstructed via their decay topology will be determined. The consistent and large acceptance in $\eta$ and $p_{\mathrm{T}}$ gives precise transverse momentum spectra for all those particle types. Through statistical model fits to this data, the freeze-out temperature and baryochemical potential of the produced system will be extracted, placing various datasets on the phase diagram of QCD, allowing the study of variation of their location with collision system size, collision energy and centrality. Secondary vertex reconstruction, threedimensional tracking of trajectories curved in a magnetic field as well as a combination of particle identification techniques will allow for the reconstruction of particles with non-zero strangeness, including multi-strange baryons such as $\Xi$ and $\Omega$, as well as hypernuclei. This is instrumental in the determination of the degree of thermalization in the system, the appearance of the deconfined state and the search for signatures of the critical point.

Strongly decaying resonances, such as $\rho(770), \mathrm{K}^{*}(892), \phi(1020), \Sigma(1385)$, and $\Lambda(1520)$ will be reconstructed with the invariant mass technique. Their abundances are sensitive to the existence and duration of the hadronic rescattering phase [4, 5], as their lifetime is comparable with the duration of this phase, in which they can undergo rescattering and regeneration. In thermal models they will also provide independent constraints of the fits.

The system created in heavy-ion collisions experiences extreme pressure gradients, which are converted into significant acceleration and collective behavior of matter. The efficiency of this conversion is directly sensitive to fundamental transport properties of matter, such as shear and bulk viscosity, as well as to the Equation of State of QCD matter. The detailed differential study of flow is therefore of utmost importance. The radial flow manifesting in common velocity for all particles pointing away from the central axis of the source modifies strongly the inclusive transverse momentum spectra of particles with different mass, from which its measurement can be inferred. The spectator nucleons are moving close to the created system in the initial stage after the collision, preventing the flow of matter in their direction. This produces the so-called directed flow $v_{1}$. At RHIC BES energies [6] it has intriguing non-monotonic behavior, which will be investigated in MPD. For non-central collisions in plane transverse to the beam direction the cross-section of the system has an almond shape, leading to the development of elliptic flow $v_{2}$. Its measurement, allows for determination of crucial properties of the system, and may reveal signatures of changes to the speed of sound $c_{s}$ near the phase transition, resulting in the softening of the EOS.

The non-monotonic increase in the fluctuations of conserved quantities is expected in the vicinity of the critical point. Event-by-event fluctuations of total event multiplicity, mean transverse momentum and conserved charges, as well as multiplicities of identified hadrons will be measured in MPD. The advantage of the collider experiment is its large acceptance (at least $|\eta|<1.2$ and $0.2<p_{\mathrm{T}}<3.0$ for identified particles), which is also constant with respect to collision energy. This presents favorable conditions for fluctuation measurements, that is low systematic uncertainties, some of which can additionally cancel out in the comparison between measurements at varying $\sqrt{s_{\mathrm{NN}}}$.

The geometrical size of the system created in heavy-ion collisions as well as important aspects of its dynamics are accessible via the femtoscopy technique. It is based on twoparticle correlation measurements, routinely carried out for pairs of identical mesons and baryons. Their study as a function of collision energy, centrality and pair type is planned in MPD. In particular, it has been argued [7], that a large ratio of system sizes in the transverse plane can be viewed as a signature of the existence of the first order phase transition. Recently the correlation measurements for pairs of baryons have been used for the determination of strong interaction between them. This is especially important for baryons with non-zero strangeness, where details of such interaction have crucial influence on the properties of dense 
nuclear matter, found in the cores of neutron stars. Baryon production will be significantly enhanced at NICA energies, making MPD particularly well suited for such measurements.

The ECal will measure electromagnetic probes at NICA. The study of direct photons at low to intermediate $p_{\mathrm{T}}$ will give information on the initial temperature of the system [8-10]. Identification of $\pi^{0}$ and $\eta$ mesons as a function of centrality and $p_{\mathrm{T}}$, will also be possible. A clean sample of electrons will be obtained by combining the energy measurement from ECal with tracking and charged particle rejection from TPC and ToF. This will allow a study of light vector mesons, as well as extensive investigation of the di-lepton production at a wide range of invariant mass, as well as the total transverse momentum of the pair. NICA offers a favorable conditions for such studies, as background is expected to be significantly reduced due to suppressed charm production.

\section{Summary}

The NICA Accelerator Complex has been briefly introduced with a report on the status of construction and commissioning of the new elements. The ion sources, as well as Nuclotron and Booster are operational. First physics run of the collider is expected in 2023. The activities in the MPD Hall are ongoing with magnet assembly well advanced. Production of subdetector components for TPC, ToF, ECal, FHCal and FFD is ongoing. Commissioning of the MPD setup expected to start in 2022.

The physics program of MPD for initial datataking has been briefly discussed. Measurements of collision centrality and event plane, together with tracking and particle identification are foreseen. Characterization of the particle production in the soft regime is the main focus, with an important addition of electromagnetic probes. MPD will perform a search for the signatures of the deconfined matter, the existence and nature of the phase transition to ordinary hadronic matter, as well as possible existence of a critical point in the phase diagram of the QCD matter. The international collaboration of MPD is preparing for first physics runs in 2023.

\section{Acknowledgments}

Author is supported by the Polish NCN grant 2017/27/B/ST2/01947, WUT ID-UB and the grants of the Polish Plenipotentiary for JINR.

\section{References}

[1] C. Loizides, J. Nagle, P. Steinberg (2014), [SoftwareX1-2,13(2015)]

[2] K. Aamodt et al. (ALICE Collaboration), Phys. Rev. Lett. 105, 252301 (2010)

[3] R. Sahoo, A.N. Mishra, N.K. Behera, B.K. Nandi, Adv. High Energy Phys. 2015, 612390 (2015), [Erratum: Adv. High Energy Phys. 2017, 4517153 (2017)]

[4] S. Acharya et al. (ALICE), Phys. Rev. C99, 064901 (2019)

[5] B.I. Abelev et al. (STAR), Phys. Rev. Lett. 97, 132301 (2006)

[6] L. Adamczyk et al. (STAR), Phys. Rev. Lett. 120, 062301 (2018)

[7] P. Batyuk, I. Karpenko, R. Lednicky, L. Malinina, K. Mikhaylov, O. Rogachevsky, D. Wielanek, Phys. Rev. C 96, 024911 (2017)

[8] A. Adare et al. (PHENIX), Phys. Rev. Lett. 104, 132301 (2010)

[9] A. Adare et al. (PHENIX), Phys. Rev. C91, 064904 (2015)

[10] J. Adam et al. (ALICE), Phys. Lett. B754, 235 (2016), 1509. 07324 